ARTICLE

\title{
How machine learning can help select capping layers to suppress perovskite degradation
}

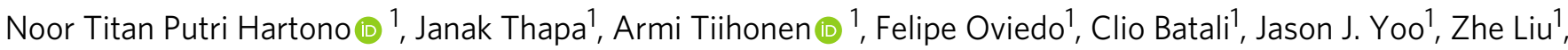 \\ Ruipeng $\mathrm{Li}^{2}$, David Fuertes Marrón ${ }^{1,3}$, Moungi G. Bawendi (i) ${ }^{1}$, Tonio Buonassisi (iD ${ }^{1 凶} \&$ Shijing Sun (D) ${ }^{1 凶}$
}

Environmental stability of perovskite solar cells (PSCs) has been improved by trial-and-error exploration of thin low-dimensional (LD) perovskite deposited on top of the perovskite absorber, called the capping layer. In this study, a machine-learning framework is presented to optimize this layer. We featurize 21 organic halide salts, apply them as capping layers onto methylammonium lead iodide $\left(\mathrm{MAPbl}_{3}\right)$ films, age them under accelerated conditions, and determine features governing stability using supervised machine learning and Shapley values. We find that organic molecules' low number of hydrogen-bonding donors and small topological polar surface area correlate with increased $\mathrm{MAPbl}_{3}$ film stability. The top performing organic halide, phenyltriethylammonium iodide (PTEAI), successfully extends the $\mathrm{MAPbl}_{3}$ stability lifetime by $4 \pm 2$ times over bare $\mathrm{MAPbl}_{3}$ and $1.3 \pm 0.3$ times over state-of-the-art octylammonium bromide $(\mathrm{OABr})$. Through characterization, we find that this capping layer stabilizes the photoactive layer by changing the surface chemistry and suppressing methylammonium loss.

\footnotetext{
${ }^{1}$ Massachusetts Institute of Technology, 77 Massachusetts Avenue, Cambridge, MA 02139, USA. ${ }^{2}$ National Synchrotron Light Source II, Brookhaven National Laboratory, Upton, NY 11973, USA. ${ }^{3}$ Instituto de Energía Solar-ETSIT, Universidad Politécnica de Madrid, 28040 Madrid, Spain.

凶email: buonassisi@mit.edu; shijings@mit.edu
} 
erovskite solar cell (PSC) stability is still far less than the $\sim 25$ years required to enter the mainstream photovoltaic (PV) market $^{1}$, despite efficiencies reaching $25.2 \%^{2}$. Improving environmental stability is a critical step. Recent studies suggest that mixing low-dimensional (LD) perovskite with the absorber improves the stability, but device performance suffers because carrier transport is reduced due limited carrier mobility through the LD material ${ }^{3-5}$.

In contrast, the capping-layer method improves poor carrier transport by intercalating the $2 \mathrm{D}$ perovskite with conductive organic materials. As a result, short-circuit current $\left(J_{\mathrm{SC}}\right)$ recovers. With improved surface passivation because of the capping layer, the open-circuit voltage $\left(V_{\mathrm{OC}}\right)$ increases, as does environmental stability at ambient temperature with elevated (40-90\%) relative humidity $(\mathrm{RH})^{6,7}$. The capping layer is formed by reacting organic halides in a solvent with the $3 \mathrm{D}$ perovskite network underneath, forming the LD perovskite network with intercalated organics. The choice of organic halides is known to affect device stability, however the relationship between structure and stability has not been fully explored, in part because the parameter space is vast ${ }^{8}$. Perovskite thin-film deposition with a range of organic halides has been reported, including benzene rings/phenyl with amine (e.g., phenylethylammonium iodide ${ }^{9-11}$, phenylethylammonium bromide ${ }^{9,12}$, aniline iodide ${ }^{13,14}$, benzylammonium iodide $^{14}$, teophylline ${ }^{15}$, caffeine ${ }^{16}$ ), long carbon chains with amine (e.g., $n$-butylammonium iodide ${ }^{17,18}, n$-octylammonium iodide $\left.^{18}\right)$, fluorous amine (e.g., 2-(4-fluorophenyl)ethylammonium iodide ${ }^{19}$ ), branched amines (e.g., 1,8-octanediammonium iodide $^{20}$, diethylammonium bromide ${ }^{21}$, diethylammonium iodide $^{21}, n$-hexyl trimethyl ammonium bromide ${ }^{6}$ ), and large, complex structures (e.g., Eu-porphyrin complex ${ }^{22}$ ). Photovoltaic devices based on these materials demonstrated improved stability and efficiency than their non-capped controls under various environmental test conditions. However, little is known about which specific chemical properties among the different types of organic halides control the improved stability of the capped materials.

Inspired by recent studies on inverse design of polymers and inorganic solids ${ }^{23-25}$, as well as on using machine learning to understand PSCs' properties ${ }^{26-28}$, we present a machine-learning framework to investigate LD organic-inorganic perovskites serving as a capping layer for $\mathrm{MAPbI}_{3}$. We elucidate which properties of capping layers are responsible for enhancing stability, and the underlying mechanisms whereby they work. With this information, we can generate materials-design guidelines.

\section{Results}

Study overview and objectives. We consider 21 organic salts as capping-layer materials, with different sizes, branches, and chemical properties, including both iodine and bromine-based salts. Capping layers are deposited using spin coating atop $300 \mathrm{~nm}$ thick films of methylammonium lead iodide $\left(\mathrm{CH}_{3} \mathrm{NH}_{3} \mathrm{PbI}_{3}\right.$, or $\left.\mathrm{MAPbI}_{3}\right)^{29}$. The poor $\mathrm{MAPbI}_{3}$ stability guarantees a strong baseline degradation rate, and strong signal-to-noise for our study. (In principle, the framework developed in this study can be extended to different perovskite absorber compositions, including mixed-cation and -anion materials that gained popularity in recent years.) For each film, 12 different processing conditions are explored. Following sample fabrication, perovskite films are tested unencapsulated under rigorous accelerated aging conditions (85\% $\mathrm{RH}, 85^{\circ} \mathrm{C}$ temperature, and 0.16 Sun illumination). We photograph the samples in situ every $3 \mathrm{~min}$, calibrate color using calibration tiles with thin-plate spline color warping method ${ }^{30}$, and extract numerical values for degradation onset and rate from the time series images as a proxy for film stability $28,31-33$.
To determine which capping-layer properties and processing conditions govern film stability, we employ a supervised-learning algorithm with a feature importance ranking. As model inputs, we include structural and chemical features of the organic molecules in the capping layers, derived from the PubChem $2019^{34}$ database, as well as 12 processing conditions. The 12 processing conditions vary capping-layer annealing temperature and capping-layer precursor solution concentration. As model output, we use degradation onset and rate. We then determine the feature importance ranking, using Shapley value concept ${ }^{35}$, and use this ranking to infer design rules for organic molecules comprising capping layers.

The model trained on our experimental data, and subsequent feature importance ranking, indicate that the number of hydrogen-bond donors and the organic-molecule topological surface area are the two most important features of an organic capping-layer molecule governing film stability. To determine why our best-performing molecule exhibits the best stability among the 21 screened materials, we perform in-depth materials characterization, examining both the surface and the bulk.

Figure 1a shows the overview of the study and objectives for finding the design guidelines of capping layer for suppressing degradation in perovskite solar cells. Figure $1 \mathrm{~b}$ shows the example of average change in color in the accelerated aging test, from black to yellow.

a
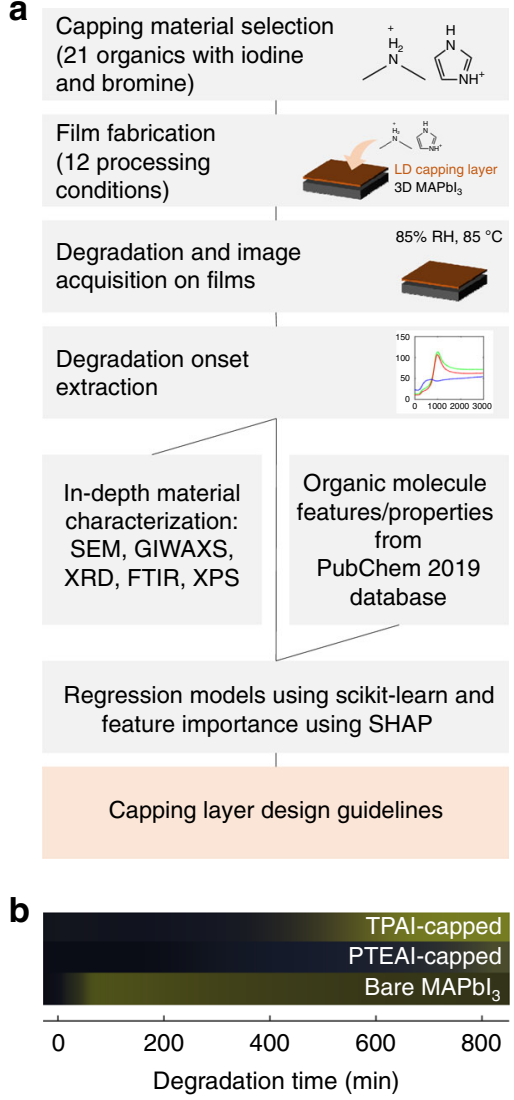

Fig. 1 The workflow of the study and aging test result over time.

a Schematic overview of this study, aimed at developing design rules for capping layer of perovskite solar cells. $\mathbf{b}$ the raw image changes for tetrapropylammonium iodide (TPAI)-capped, phenyltriethylammonium iodide (PTEAI)-capped, which have similar molecular weights, and bare $\mathrm{MAPbl}_{3}$ films. 
a
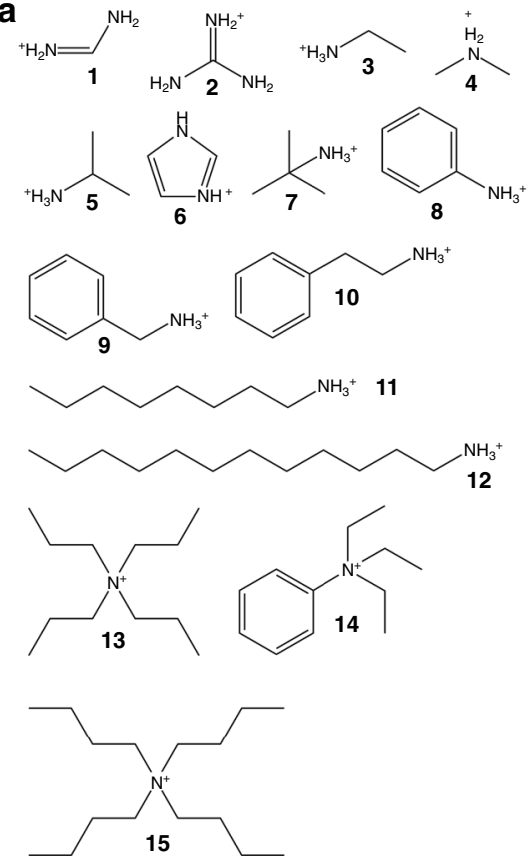

b

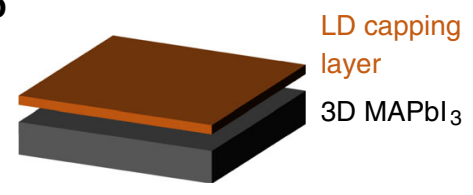

C

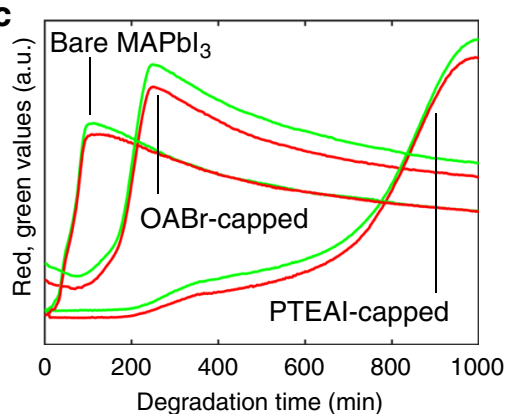

d Bare $\mathrm{MAPbl}_{3} \mathrm{OABr}$-capped PTEAl-capped $0 \mathrm{~min} 500 \mathrm{~min} 0 \mathrm{~min} 500 \mathrm{~min} 0 \mathrm{~min} 500 \mathrm{~min}$

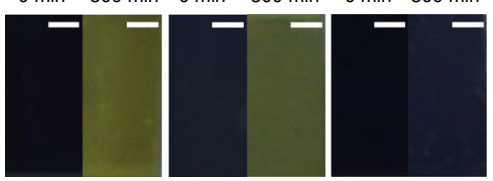

Fig. 2 Capping-layer precursors and accelerated aging results of best-performing capping-layer material. a $A$-site cations used in this study and their chemical structure: (1) formamidinium (FA), (2) guanidinium (GA), (3) ethylammonium (EA), (4) dimethylammonium (DMA), (5) iso-propylammonium (iPA), (6) imidazolium (ID), (7) t-butylammonium (tBA), (8) phenylammonium (PhA), (9) benzylammonium (BzA), (10) phenethylammonium (PEA), (11) n-octylammonium (OA), (12) dodecylammonium (DA), (13) tetrapropylammonium (TPA), (14) phenyltriethylammonium (PTEA), and (15)

tetrabutylammonium (TBA). $\mathbf{b}$ The film structure with 3D MAPbl 3 at the bottom and LD capping layer deposited atop. $\mathbf{c}$ The time-dependent red and green values of camera images, and $\mathbf{d}$ the camera images of pre-degraded and 500 min-degraded films for bare $\mathrm{MAPbl}_{3} \mathrm{control}_{\mathrm{material}}$, (11) $\mathrm{OABr}$, used in state-of-the-art high-efficiency devices, and (14) PTEAI, our best-performing material in this study, with scale bar $0.5 \mathrm{~cm}$.

Capping-layer composition, fabrication, and aging tests. Cappinglayer precursors consist of $15 A$-site organic cations in different lengths and shapes (Fig. 2a). The number of carbon atoms in these materials ranges between 1 and 16, with primary (two $\mathrm{N}-\mathrm{H}$ bonds), secondary (one $\mathrm{N}-\mathrm{H}$ bond), tertiary (no $\mathrm{N}-\mathrm{H}$ bond) amine, or quaternary (no $\mathrm{N}-\mathrm{H}$ bond, no lone pairs). Two $X$-site anions, iodide and bromide, are tested. A total of 21 unique organic-halide salts are explored. The $A X$ capping-layer material is dissolved in solvent, spin coated atop a $\mathrm{MAPbI}_{3}$ thin film with excess $\mathrm{PbI}_{2}{ }^{36}$ (Fig. 2b), and annealed at temperatures between 50 and $125^{\circ} \mathrm{C}$ for $10 \mathrm{~min}$.

For the samples that stay dark for longer than $300 \mathrm{~min}$, each sample condition is repeated for at least two times to ensure adequate statistics. The samples were aged under $85 \% \mathrm{RH}, 85^{\circ} \mathrm{C}$ temperature, and 0.16 Sun visible-only illumination in batches of 28 samples. Each aging test is stopped after the sample turns yellow, indicating the degradation from perovskite black phase into the $\mathrm{PbI}_{2}$ phase ${ }^{37,38}$. Illumination $(0.16 \mathrm{Sun})$ is added, which allows optical images to be taken every $3 \mathrm{~min}$ as a proxy for film degradation. The red, green, and blue (RGB) color values are extracted for different time points of the degradation from the image data. The increasing red and green (RG) colors correspond to changes in film color from black to yellow, as shown in Fig. 2c, $\mathrm{d}$. The onsets are described as the rapid change from black to yellow evidenced in the red and green (RG) channels (Supplementary Figs. 1 and 2), which are the key descriptors for stability in this study. Although this study focuses on the degradation onset, it is possible to consider the rate of color change (or the slope, Supplementary Fig. 3). Because both red and green channels overlap, it is sufficient to consider only the red channel (Supplementary Fig. 4). The complete comparison of film color before and during the degradation is shown in Supplementary
Fig. 2, where the bare $\mathrm{MAPbI}_{3}$ color change onset occurred at $107 \mathrm{~min}$ on average, and $\mathrm{MAPbI}_{3}$ films with specific capping layers, e.g., tetrapropylammonium iodide (TPAI), tetrapropylammonium bromide (TPABr), tetrabutylammonium iodide (TBAI), tetrabutylammonium bromide (TBABr), and phenyltriethylammonium iodide (PTEAI) retained their dark color $4 \pm 2$ times longer. The longer alkyl chain performs better than the shorter one. Branched molecule and phenyl group molecule also lead to better film stability. All the most stable capping-layer materials in this study have quaternary ammonium group, instead of primary, secondary, or tertiary. The quaternary ammonium has been shown to effectively passivate the charged defects and help to minimize the initiation of film degradation ${ }^{39}$.

Machine-learning regression, feature importance ranking, and design principles. Machine-learning regression is performed on a color change-based degradation descriptor described in the previous section. Colors of the samples are extracted from JPEG pictures that have been color calibrated to ensure reproducibility and repeatability. Specifically, the onsets, i.e., time-intercepts of rapid color change from black to yellow, are the key descriptors for stability in this study. The onset time is a continuous variable; hence we are using regression as supposed to classification machine-learning models. This onset descriptor acts as the output of our machine-learning model, which is used to train the models with the input coming from the database and the processing condition.

We featurize 21 organic capping-layer materials using their material properties from the PubChem 2019 database ${ }^{34}$, namely molecular weight, $x \log P$ (or partition coefficient that indicates hydrophobicity/hydrophilicity of molecules $)^{40}$, the number of 
a

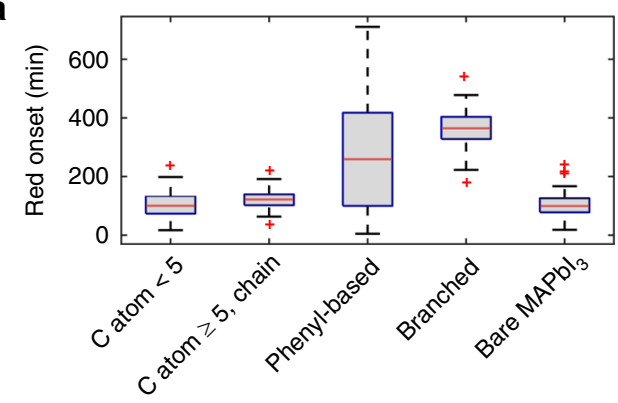

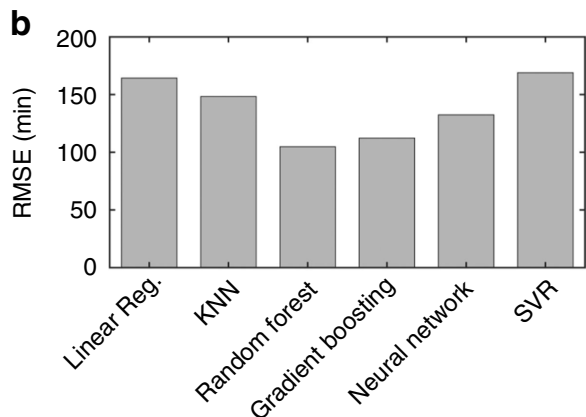

d
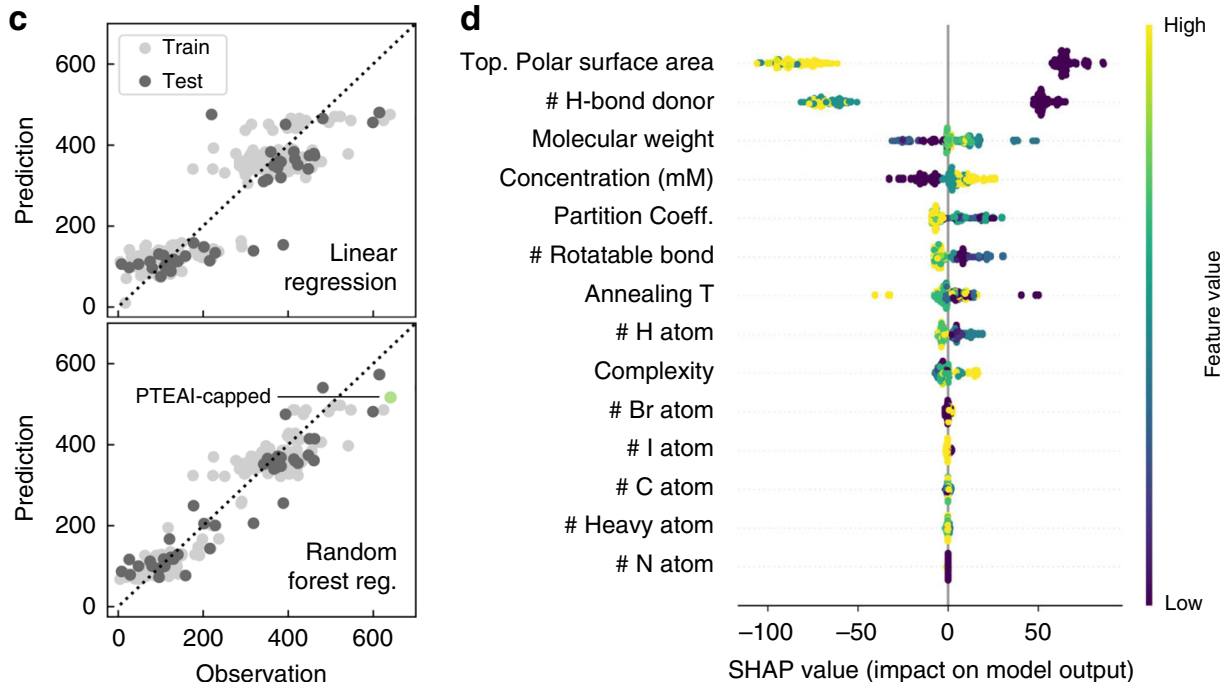

Fig. 3 Image data parameter extraction, regression results, and Shapley-based importance rank. a Extracted red onset points (in minutes) and their distributions from time-dependent camera data of capping-layer groups and bare $\mathrm{MAPbl}_{3}$ controls, synthesized using 12 different processing conditions. The red line shows the median, and the box shows the upper and lower quartiles. $\mathbf{b}$ The cross-validated root mean square error (RMSE) of various machine-learning models, including linear regression, K-nearest neighbors (KNN), random forest regression, gradient boosting regression with decision trees, neural network (multilayer perceptron), and support vector regression (SVR), with non-normalized input. c The comparison of red onset prediction and observation output between linear regression and random forest regression, with the green circle indicating PTEAI-capped film. $\mathbf{d}$ The feature importance ranking obtained from the random forest regression algorithm and SHAP library, showing the chemical properties and processing conditions in descending order of importance (rank). The yellow and purple color indicates high and low values of a given feature, respectively.

rotatable bonds, complexity ${ }^{41,42}$, topological polar surface area $(\mathrm{TPSA})^{43}$, the number of hydrogen-bond donors ${ }^{44}$, and the number of each element (carbon, hydrogen, bromine, nitrogen, and iodine). For each of the 21 candidate materials, we test 12 unique processing conditions by varying the annealing temperature of capping layer after spin coating step, and the concentration of capping-layer precursor solution. These material-property and processing-condition features are used as inputs into our machine-learning models, and are described in more detail in Supplementary Table 1 . The 21 capping-layer materials can be grouped arbitrarily into four different groups: organics with carbon atom fewer than 5, long-chain organics with carbon atom more than 5, phenyl-based organics, and branched organics, as shown in Fig. 3a. In general, both phenyl-based ${ }^{14}$ and branched ${ }^{45}$ organics have previously been reported to increase stability.

Using the scikit-learn library in Python ${ }^{46}, 6$ regression models are trained on the data from 260 accelerated aging tests shown in Fig. 3a, including linear regression, K-nearest neighbors (KNN) regression ${ }^{47}$, random forest regression ${ }^{48}$, gradient boosting regression with decision trees ${ }^{49}$, multilayer perceptron neural network with three hidden layers (each has 128, 256, and 64 units) by using Adam solver ${ }^{50}$, and support vector machine regression $(\mathrm{SVR})^{51}$. The hyperparameters in different models are optimized (Supplementary Tables 2 and 3) using GridSearchCV function on scikit-learn library, which performs an exhaustive search of parameters based on minimum fivefold cross-validated root mean squared error (RMSE). The ML input is either normalized, which calibrates the mean to zero and scales to unit variance, or not. The fivefold cross-validated RMSE result for both input types are shown in Supplementary Fig. 5. Random forest regression results in the lowest fivefold cross-validated RMSE for non-normalized input, as shown in Fig. 3b. Random forest regression is a method with several decision trees, where each of their estimators is independently predicted from a different subset of data, and in the end, the estimators are averaged $^{52}$. The RMSE of linear regression is quite high and it has inconsistent weights (as shown in Supplementary Figs. 6 and 7). The RMSE of multilayer perceptron neural network is large due to dataset that is small to be used with neural-network method. RMSE's of the random forest regression and gradient boosting regression with decision trees are lower, about 104 and $112 \mathrm{~min}$, respectively, which is still high, considering the degradation onset range of $0-700 \mathrm{~min}$. The high RMSE is caused by the variability in 12 different synthesis conditions, in addition to inherent high variability in the bare $\mathrm{MAPbI}_{3}$ 's degradation profile $\mathrm{e}^{53,54}$ (standard deviation of red onset across 35 bare $\mathrm{MAPbI}_{3}$ samples $\approx 45 \mathrm{~min}$ ), as shown in Fig. $3 \mathrm{a}$ and Supplementary Table 4 . Figure $3 \mathrm{c}$ demonstrates the randomly-split 20\%:80\% test:train set 


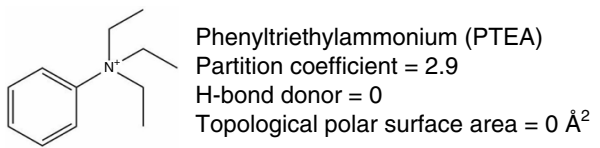

b

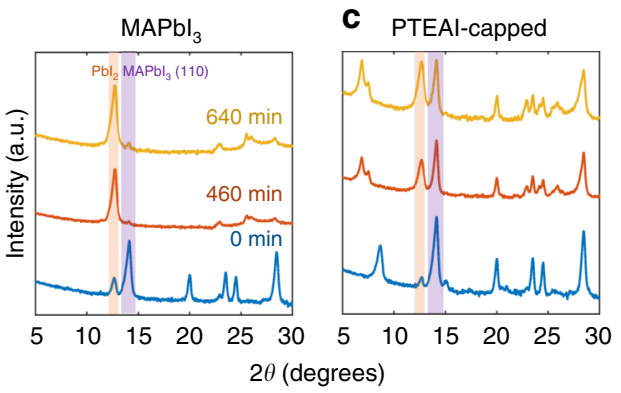

d

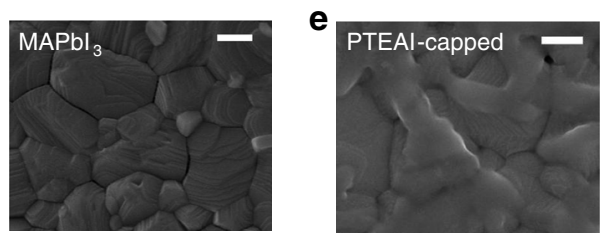

$\mathbf{f}$

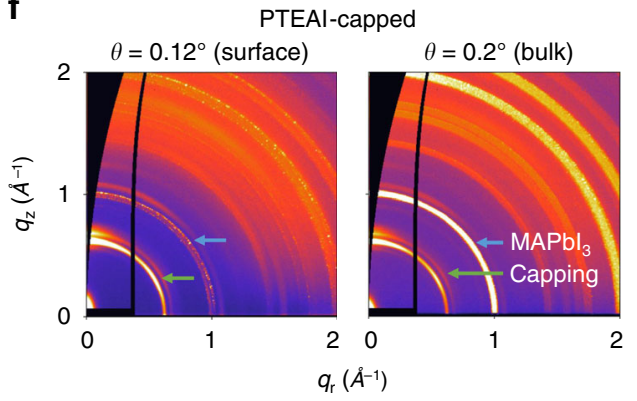

Fig. 4 Crystal structure and morphology characterization for optimum capping layer (PTEAI). a Phenyltriethylammonium (PTEA) has 0 hydrogen-bond donor due to its quaternary amine. Powder $X$-ray diffraction (XRD) spectra of bare (b) and PTEAl-capped $\mathrm{MAPbl}_{3}(\mathbf{c})$, when they were degraded for 460 and 640 min The purple shaded area indicates the (110) peak of $\mathrm{MAPbl}_{3}$, and the orange shaded area indicates the (001) $\mathrm{Pbl}_{2}$ peak. Pre-degraded scanning electron microscopy (SEM) image of bare (d) and PTEAl-capped $\mathrm{MAPbl}_{3}$ (e), scale bar: $200 \mathrm{~nm}$. f Grazing-incidence wideangle $x$-ray scattering (GIWAXS) images of PTEAI-capped $\mathrm{MAPbl}_{3}$ presenting the structure on the surface and in the bulk films, with the incident angles of $0.12^{\circ}$ and $0.2^{\circ}$, respectively. The green arrow points to LD capping peak, and the blue arrow points to the $\mathrm{MAPbl}_{3}$ peak.

observation and prediction results of random forest regression and linear regression, which shows that the random forest model is the best-performing model for our data, without the evidence of overfitting.

To interpret our model, we further analyze the random forest regression results using SHAP (Shapley Additive exPlanations) ${ }^{35}$, a generalized metric for feature importance, which utilizes the game-theory-based Shapley values to calculate the contribution of each feature to the model's output. SHAP indicates how each feature contributes to the model output (red color change onset time). Figure $3 \mathrm{~d}$ shows the 14 features (inputs) ranked using SHAP based on random forest regression. The yellow color corresponds to high value of the features consisting of molecular properties and processing conditions, whereas the purple color corresponds to low value of the features. The $x$-axis of Fig. $3 \mathrm{~d}$, labeled as the SHAP value (impact on the model output), represents the red degradation onset values. If the SHAP value is positive, the degradation onset increases hence the film lasts longer, and vice versa. The $y$-axis of Fig. 3d, listing all the 14 features, are ranked based on their contribution to the degradation onset.

Low number of hydrogen-bond donor and small topological polar surface area (TPSA) are the top most important factors determining stability, shown as the yellow points on the positive side of SHAP value. PTEAI, as the most stable capping-layer material in this study, indeed has a low number of hydrogenbond donor (0) and a small TPSA $\left(0 \AA^{2}\right)$. The statistical analysis (ANCOVA) of the films shows that the red degradation onset of the most stable capping-layer material in this study, PTEAI, is statistically significantly different, with $95 \%$ confidence level, in comparison to other materials and bare $\mathrm{MAPbI}_{3}$ film (Supplementary Fig. 8). This result is also consistent with gradient boosting regression with decision trees method (Supplementary Fig. 9). Both number of hydrogen-bond donor and TPSA are correlated, because the hydrogen-bond donor presents when there is a bond between electronegative atoms (in our case, nitrogen) and hydrogen, creating a polar surface area on the molecule. The Pearson correlation value coefficient of hydrogenbond donor and TPSA is 0.81 (Supplementary Fig. 10) ${ }^{43}$. This evidence might support the hypothesis that hydrogen bonding plays a very important role in degradation of perovskite solar cells ${ }^{55-59}$, especially under high-humidity testing conditions. The next important features which affect stability are molecular weight and concentration of precursor solution. On the other hand, $x$ log $P$ or partition coefficient (an indicator for hydrophobicity), complexity, and the number of carbons, iodines, or bromines generally have lower ranks in the model. If we consider the recently published capping-layer materials, such as theophylline, caffeine, and theobromine ${ }^{15,16}$, assuming they were fabricated and aged in the same manner as the materials in this study, the onsets are predicted to happen at 103.2, 264.2, and $121.5 \mathrm{~min}$ respectively. As a reference, PTEAI onset happens at $(462 \pm 115)$ min. Due to its lower number of hydrogen-bond donor and smaller polar surface area, caffeine is predicted to be more stable among these three materials, even though its surface passivation property is worse than theophylline ${ }^{15}$. If the topological polar surface area and number of hydrogen bonding are indeed the most important features, in future studies researchers can explore more complex quaternary ammonium group $\left(\mathrm{NR}_{4}{ }^{+}\right)$, where $\mathrm{R}$ is an alkyl or an aryl group, for instance, $N, N, N$-trimethylnaphthalen-1-aminium iodide (Supplementary Fig. 11).

The advantage of using SHAP instead of traditional interpretability methods, is its robustness to correlated features ${ }^{35}$ than traditional interpretability methods, due to the game-theoretic nature of feature attribution. However, if strong multicollinearity exists (i.e., very high shared variance among features causing significant variations in model training), highly correlated features, for instance the molecular weight and the number of carbon atoms, might be incorrectly attributed feature importance, according to how the model is fitting the data. To diminish these spurious attributions, we train various different models via crossvalidation and make our final decision based on contrasting the feature ranks (Supplementary Figs. 12-18). The consistency among models, along with testing using cross-validation, allows us to physically interpret the models. The most robust approach, which is incompatible with our exploratory goals, could have been to perform causal inference using randomized control trials or experiments in a more limited compound space. The tradeoff, of course, is the slow and poor exploration of the material space.

Protection mechanisms in top-performing candidates. As suggested by feature importance ranking, our top-performing capping-layer material, PTEAI, which is reported to have no 
hydrogen bonding acceptor and $0 \AA^{2}$ of TPSA as shown in Fig. 4a, does achieve high stability ${ }^{34}$. The PTEAI-capped perovskite enables $1.3 \pm 0.3$ times and $4 \pm 2$ times improvement in stability, to the state-of-the-art $\mathrm{OABr}$ capping layer that we test in this study, and bare $\mathrm{MAPbI}_{3}$ films, respectively ${ }^{7}$. To better understand the protection mechanisms achieved by the PTEAI capping layer, we further compared PTEAI-capped perovskite with other capping layers such as TPAI, which contains the same number of carbon atoms as PTEAI and has a similar molecular weights but degrades faster, via X-ray diffraction (XRD), scanning electron microscopy (SEM), and grazing-incidence wide-angle-Xray scattering (GIWAXS), as shown in Supplementary Figs. $19-26$.

The powder XRD data are compared to butylammonium (BA)based 2D perovskite to confirm the new phase ${ }^{60-62}$. The addition of a capping layer indeed introduces a new phase in the film, indicated by the emergence of a new peak at $8.68^{\circ}$ in the case of a PTEAI capping layer, which matches with $(B A)_{2}(\mathrm{MA})_{3} \mathrm{~Pb}_{4} \mathrm{I}_{13}$, as shown in Fig. $4 \mathrm{c}$ and Supplementary Fig. 19. This indicates a Ruddlesden-Popper (RP) perovskite phase, (PTEA) $)_{2}(\mathrm{MA})_{3} \mathrm{~Pb}_{4} \mathrm{I}_{13}$ $(n=4)^{60-62} . n$ indicates the number of $3 \mathrm{D}$ perovskite layers before separated by organic molecules, and the RP perovskite formula is in the form of $A_{2}^{\prime} A_{n-1} B_{n} X_{3 n+1}$, where $A, B$, and $X$ correspond to $A$-site cation, $B$-site cation, and $X$-anion. In the case of the TPAI capping layer, the new peak matches with $(\mathrm{BA})_{2}(\mathrm{MA})_{2} \mathrm{~Pb}_{3} \mathrm{I}_{10}$, indicating a RP perovskite phase, (TPA $)_{2}(-$ $\mathrm{MA})_{2} \mathrm{~Pb}_{3} \mathrm{I}_{10}(n=3)$, as shown in Supplementary Fig. 2060-62. The capping layer reacts with the excess $\mathrm{PbI}_{2}$ coming from the $\mathrm{MAPbI}_{3}$ layer underneath, forming the RP perovskite phase at the top ${ }^{7}$.

Within 460 min of accelerated aging tests, almost all of the bare $\mathrm{MAPbI}_{3}$ degrades into lead iodide $\left(\mathrm{PbI}_{2}\right)$, as indicated by the emergent peak at $12.64^{\circ}$ and almost complete suppression of the $\mathrm{MAPbI}_{3}$ related peak at $14.02^{\circ}$, highlighted by the shaded purple area in Fig. 4b. The PTEAI-capped films, on the other hand, maintains its $\mathrm{MAPbI}_{3}$ and RP peaks (Fig. 4c), albeit shifted due to thermally induced structural modification ${ }^{63}$, much longer than bare or TPAI-capped films, shown in Supplementary Fig. 21. This is further evidence that the RP phase in the capping layer reduces the $\mathrm{MAPbI}_{3}$ degradation rate, because it helps suppress the conversion of $\mathrm{MAPbI}_{3}$ into $\mathrm{PbI}_{2}$.

Comparing the surface morphology of $\mathrm{MAPbI}_{3}$ and PTEAIcapped $\mathrm{MAPbI}_{3}$, we noticed a difference at pre-degradation time point, where the capping layers coat the surface of $\mathrm{MAPbI}_{3}$, including the grain boundaries, as shown in Fig. 4d, e. As degradation occurs, and the surface reacts with the high-humidity environment at elevated temperature, the grains change and more pinholes appear, as shown in Supplementary Fig. 22.

GIWAXS images provide a deeper understanding about the crystal structure difference of the surface with respect to the bulk of the films. Figure $4 \mathrm{f}$ shows the pre-degraded data for the surface at low incidence angle $\left(\theta=0.12^{\circ}\right)$ below the critical angle of perovskites $\left(0.18^{\circ}\right)$, and the bulk at higher incidence angle $(\theta=$ $0.2^{\circ}$ ) along $q_{\mathrm{r}}$ (horizontal) and $q_{\mathrm{z}}$ (vertical) axes. The blue arrow for capped samples shows the signature of capping materials/RP phase, whereas the green arrow shows the $\mathrm{MAPbI}_{3}$ phase. The ratio of the RP phase of PTEAI capping layer and the $\mathrm{MAPbI}_{3}$ phase close to the surface is much larger than deeper in the bulk, which is dominated by the $\mathrm{MAPbI}_{3}$ phase. This indicates that the capping-layer material mostly resides on the surface of the perovskite thin film. In addition, we find that the LD perovskite based on PTEAI and TPAI show different texture, based on their vertical and horizontal integration of GIWAXS data. Further analysis on crystallite textures from GIWAXS is shown in Supplementary Figs. 23-26.
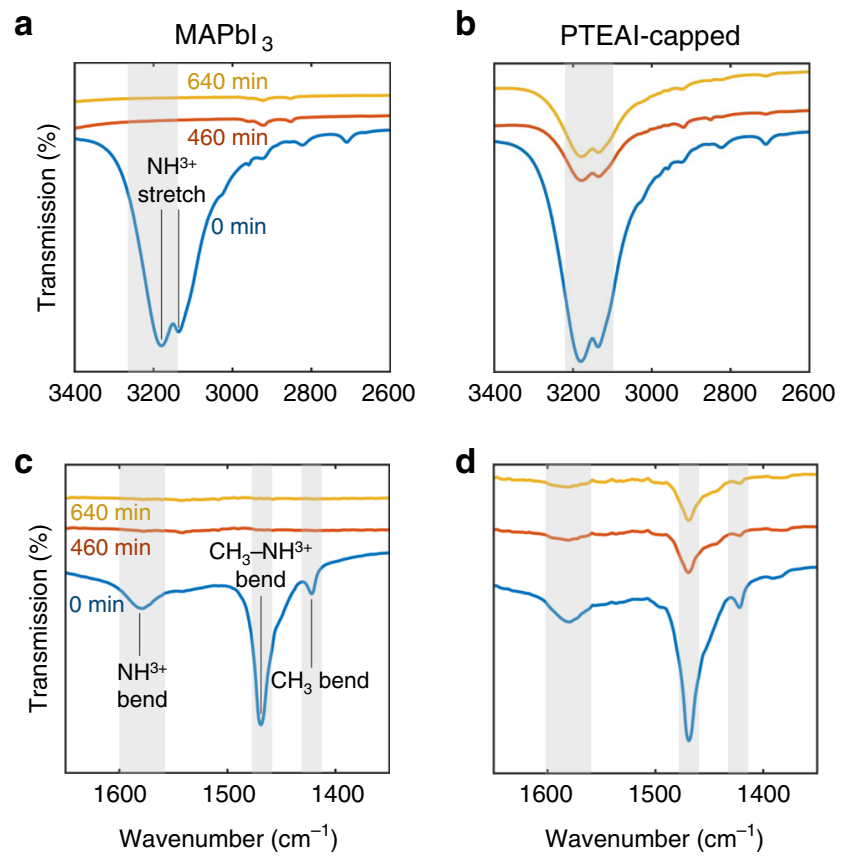

e $\mathrm{MAPbl}_{3}$

PTEAI-capped

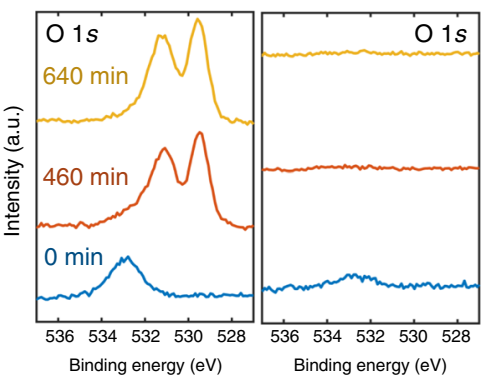

f

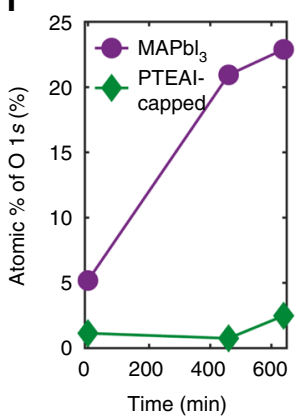

Fig. 5 Capping-layer surface chemistry characterization of optimum capping layer (PTEAI). Attenuated total reflection Fourier-transform infrared spectroscopy (ATR-FTIR) with ZnSe crystal of bare $(\mathbf{a}, \mathbf{c})$ and PTEAl-capped $\mathrm{MAPbl}_{3}(\mathbf{b}, \mathbf{d})$. e The $\mathrm{O}$ 1s spectra obtained from x-ray photoelectron spectroscopy (XPS) of bare and PTEAl-capped $\mathrm{MAPbl}_{3}$, and f their corresponding atomic percentages. The purple circle and the green diamond represent the atomic percentages of $\mathrm{O}$ 1s for bare and PTEAIcapped $\mathrm{MAPbl}_{3}$ film, respectively.

The feature importance ranking indicates hydrogen bonding donor and topological polar surface area as the most important capping-layer features, toward which the amine in the organics significantly contributes. We hence investigate the surface chemistry of the organic molecules in PTEAI-capped films. Changes in organic-molecule bonds within the perovskite films can be detected using Fourier-transform infrared spectroscopy (FTIR) attenuated total reflection (ATR) geometry with a zinc selenide (ZnSe) crystal. We measure bare $\mathrm{MAPbI}_{3}$ and PTEAIcapped $\mathrm{MAPbI}_{3}$ using FTIR, and observe the main signature of methylammonium (MA) at 3176 and $1580 \mathrm{~cm}^{-1}$, indicated by $\mathrm{NH}^{3+}$ stretch (Fig. 5a, b) and bend (Figs. 5c, d) respectively. This result suggests that MA in bare $\mathrm{MAPbI}_{3}$ disappeared at $460 \mathrm{~min}$, whereas MA in the PTEAI-capped film remained after 460 mins.

Using X-ray photoelectron spectroscopy (XPS), we observed no significant traces of oxygen (O $1 s)$ on the surface of PTEAIcapped film, even after $640 \mathrm{~min}$ of accelerated aging tests (Fig. 5e), revealing that the PTEAI-capped $\mathrm{MAPbI}_{3}$ inhibits the formation of an oxygen-containing compounds ${ }^{64}$, and increases film 
stability. In contrast, we found that the amount of oxygencontaining compounds ${ }^{64}$ in bare $\mathrm{MAPbI}_{3}$ increases within 460 min, which can be attributed to a $\mathrm{PbO}_{x}$ compound ${ }^{64,65}$. The presence of the oxygen in fresh bare $\mathrm{MAPbI}_{3}$ indicating surface contamination from atmospheric oxygen is significantly higher than in fresh PTEAI-capped samples. After fitting the C $1 s$, I $3 d$, $\mathrm{N} 1 s, \mathrm{O} 1 s$, and $\mathrm{Pb} 4 f \mathrm{XPS}$ peaks, the atomic percentages of each element are calculated; for $\mathrm{MAPbI}_{3}$, the $\mathrm{O} 1 s$ atomic composition percentage increases from about 5-20\% after aging for $460 \mathrm{~min}$, whereas for PTEAI-capped $\mathrm{MAPbI}_{3}$, the $\mathrm{O} 1 \mathrm{~s}$ atomic composition percentage stays below $1 \%$, as shown in Fig. 5 f.

Considering the inorganic materials change on the film surface, the XPS scans of $\mathrm{Pb} 4 f$ reveal the presence of two different $\mathrm{Pb}$ containing species, in the near-surface region of the capped layers, indicated by two doublet peaks, as shown in Supplementary Fig. 27 and in agreement with the formation of a second $\mathrm{Pb}$ containing species in the RP phases of both TPAI- and PTEAIcapped samples. In bare $\mathrm{MAPbI}_{3}$, the initial one doublet peak of $\mathrm{Pb} 4 f$ is at $138.8 \mathrm{eV}$, and shifts to $138.2 \mathrm{eV}$ as it degrades. The capping layers, on the other hand, initially has two doublet peaks, indicating the presence of two different $\mathrm{Pb}$-bonds on the surface. After 640 min, PTEAI-capped films still preserve its two doublet peaks, while the TPAI-capped film's extra peak disappears. The co-existence of the two distinct $\mathrm{Pb}$-doublets in capping-layer samples as a function of time can therefore be directly correlated with the resilience of the capping-layer surface to air exposure and the observed stability of the absorber material.

\section{Discussion}

In this study, we present a machine-learning-assisted investigation of the features that increase the effectiveness of hybrid organic-LD perovskite capping layers atop lead-halide perovskite solar cells. We test $\mathrm{MAPbI}_{3}$ coated with 21 combinations of organic molecules and halide anions, under accelerated aging conditions of $85 \% \mathrm{RH}, 85^{\circ} \mathrm{C}$, and 0.16 Sun illumination, and featurize the organic molecules according to open-source database values. We apply a random forest regression algorithm and SHAP values to identify which features correlate most strongly with improved stability, and find that the most important properties extending the degradation onset are (i) the low number of hydrogen-bond donors and (ii) the small topological polar surface area of the organic capping-layer molecules. By utilizing the organic salt that exhibits the strongest features, phenyltriethylammonium iodide (PTEAI), we increase the stability of bare $\mathrm{MAPbI}_{3}$ and state-of-the-art high-efficiency $\mathrm{MAPbI}_{3}$ with an $\mathrm{OABr}$-based capping layer by more than $4 \pm 2$ and $1.3 \pm 0.3$ times, respectively. Synchrotron-based XRD indicates a new Ruddlesden-Popper perovskite, (PTEA $)_{2}(\mathrm{MA})_{3} \mathrm{~Pb}_{4} \mathrm{I}_{13}$, which serves as a capping layer. XPS and FTIR reveal that the topperforming capping layer stabilizes the $\mathrm{MAPbI}_{3}$ perovskite by modifying the surface structure and chemistry, which coincides with suppression in the methylammonium loss and formation of both $\mathrm{PbI}_{2}$ and oxygen-containing compounds at the surface of perovskite. Our findings suggest capping-layer design rules that may further enhance the environmental stability of halide perovskite-based devices under harsh conditions, and pushing perovskite-based solar cells closer toward mainstream photovoltaics market.

\section{Methods}

Film and capping-layer fabrication. For $3 \mathrm{D}$ methylammonium lead iodide $\left(\mathrm{MAPbI}_{3}\right)$ precursor solution: $1.5 \mathrm{M} \mathrm{PbI}_{2}$ (TCI Chemicals) solution was dissolved in 9:1 DMF:DMSO mixed solvents, before mixing them with ammonium powder. For every gram of methylammonium iodide (MAI) powder (Dyenamo), we added $5.10 \mathrm{~mL} \mathrm{PbI}_{2}$ stock solution correspondingly, which corresponds to MAI:PbI molar ratio of 1:1.09. Capping-layer solutions were made in three different concentrations, 5,10 , and $15 \mathrm{mM}$, by mixing ammonium iodide/ammonium bromide powder with isopropyl alcohol, pure, ACS reagent, $\geq 99.5 \%$ (Sigma-Aldrich). A list of ammonium iodide/ammonium bromide powder manufacturers is listed in Supplementary Table 5.

$65 \mu \mathrm{L}$ of $\mathrm{MAPbI}_{3}$ solution was then deposited on the precleaned substrate (glass slides for UV-Vis, GIWAXS, and FTIR and XRD, FTO substrates for SEM, and XPS), and spin coated with this 2-step recipe: $1000 \mathrm{rpm}$ for $10 \mathrm{~s}$ and acceleration of $200 \mathrm{rpm} / \mathrm{s}$, then $6000 \mathrm{rpm}$ for $30 \mathrm{~s}$ and acceleration of $2000 \mathrm{rpm} / \mathrm{s}$. $5 \mathrm{~s}$ after the start of the second step, $150 \mu \mathrm{L}$ of chlorobenzene was dropped on the substrate. Then, the deposited film was annealed on the hotplate at $100^{\circ} \mathrm{C}$ for $10 \mathrm{~min}$. After the substrate is cooled down, $60 \mu \mathrm{L}$ of capping-layer solution was deposited on top, and spin coated with $3000 \mathrm{rpm}$ speed for $30 \mathrm{~s}$. The substrate was then annealed with various temperatures, $50,75,100$, and $125^{\circ} \mathrm{C}$, for $10 \mathrm{~min}$.

General characterization. The crystal structure and the film phases were characterized using X-ray diffraction (XRD, Rigaku SmartLab), with Cu-Ka sources. The film morphology and device cross-section were investigated using a ZEISS Ultra-55 field-emission scanning electron microscope (FESEM, ZEISS). The X-ray photoelectron spectroscopy was measured using K-Alpha+ XPS (Thermo Scientific) with $\mathrm{Al}-\mathrm{Ka}$ excitation source. The Fourier-transform infrared spectroscopy (FTIR) was measured using Perkin-Elmer Spectrum 400 (Perkin-Elmer), in attenuated total reflection (ATR) configuration with $\mathrm{ZnSe}$ and Ge crystals. Samples were stored in inert conditions inside a nitrogen-purged glovebox between synthesis and aging test/characterization steps.

GIWAXS characterization. Grazing-incidence wide-angle X-ray scattering (GIWAXS) measurements on perovskite thin films were taken at beamline 11-BM (CMS) at the National Synchrotron Light Source II (NSLS-II) of Brookhaven National Laboratory. The X-ray beam with the energy of $13.5 \mathrm{keV}$ shone on the thin films in the grazing incident geometry. Multiple incident angles were chosen to tune the X-ray penetration of the films and probe the structure of the surface and the bulk. The scattering spectra were collected with the exposure time of $30 \mathrm{~s}$ by an area detector (DECTRIS Pilatus $800 \mathrm{~K}$ ) placed $257 \mathrm{~mm}$ away from the sample. The data analysis was performed by using custom-made software SciAnalysis ${ }^{66}$

Accelerated aging chamber and image acquisition. The images during accelerated aging tests were acquired using a Thorlabs DCC1645C CMOS USB camera (with IR-Cut-Filter $650 \mathrm{~nm}$ removed), taken every 3 min automatically using LabView software. X-Rite Color Checker Passport 2 was used as a color reference to transform the sample images to the $\mathrm{L}^{*} \mathrm{a} * \mathrm{~b}$ color space. The color calibration used $3 \mathrm{D}$ thin-plate spline color warping method, and the resulting data were transformed back to red, green, blue (RGB) color space ${ }^{30}$. JPEG is a more compressed file format than raw bitmap BMP. The quantitative RG color values extracted from both formats, from the initial black perovskite phase until they completely degrade and turn yellow, show negligible difference (Supplementary Fig. 28). As the JPEG image data are more compressed and hence are faster to be processed, are used as the stability proxy (Fig. 2c; Supplementary Fig. 2). The relative humidity $(\mathrm{RH})$ set point was maintained at $85 \pm 3 \%$ using an Arduinocontrolled feedback system, and both the $\mathrm{RH}$ and temperature were measured using an Adafruit Si7021 sensor and EasyLog EL-USB-2 data logger. The visible only white illumination intensity in the chamber was $0.16 \mathrm{Sun}$, using an Advanced Illumination DL097 LED lamp. The samples were heated using in-house-built graphite heating elements, controlled at $85 \pm 2{ }^{\circ} \mathrm{C}$. Sample placement inside the aging chamber was randomized, to minimize risk of systematic placement-related errors. The humidity, heating element temperature, and chamber temperature were recorded throughout the test, ensuring the environment humidity and temperature profiles in each round were the same.

Data integrity. The synthesis conditions were recorded by the experimenter using a laboratory notebook, then transcribed to Google Sheets. Accelerated aging test data (camera image time series) were automatically pushed to Dropbox, and subsequently quantified using the $3 \mathrm{D}$ thin-plate spline color warping method. Features were extracted from calibrated RGB data using Python and MATLAB, where various parameters including red-channel onset (the time-intercept of red color degradation that corresponds to the yellowing/changing of perovskite phase into $\mathrm{PbI}_{2}$ ) were extracted onto a local computer. Raw GIWAXS, XPS, and FTIR data were processed using their own software packages, with different file labeling conventions, and stored on different local computers. Metadata (linking different files containing synthesis conditions, calibrated aging test data, GIWAXS, XPS, and FTIR data) were created on an ad-hoc basis, as samples were deemed of high scientific significance. Not all the data obtained are reported in this paper.

Machine-learning analysis. All the features' values are numerical and can be treated as continuous variables, which therefore do not need further encoding. The capping-layer material name is not used as one of the features, because its properties have been included in the features instead. Therefore, the preprocessing done is normalization of the input data for the models. We performed normalization of the model inputs $(X)$ using the StandardScaler algorithm, in the scikit-learn library ${ }^{46}$, which calibrates the mean and scales to unit variance. The inputs of the 
tree-based algorithms, however, do not need normalization. Therefore, we consider both the normalized/non-normalized input $(X)$, and compared their crossvalidated root mean squared error (RMSE). All the machine-learning models presented in this study were constructed using the scikit-learn library in Python. 6 machine-learning models are trained, including ordinary least squares (LR), Knearest neighbor regression $(\mathrm{KNN})$, random forest regression $(\mathrm{RF})$, gradient boosting regression with decision trees (GB), neural network (multilayer perceptron) regression $(\mathrm{NN})$, and support vector machine regression (SVR). The LR model minimizes the residual sum of squares between the experimental/observed data points and the predicted data points (MSE). This serves as the benchmark of the other non-linear 5 algorithms. The other 5 algorithms have their hyperparameters optimized using GridSearchCV function based on their MSE after fivefold cross-validation (random $80 \%: 20 \%$ training: test split). The cross-validation approximates the testing error, or the error of generalization to related out-ofdistribution data. The model hyperparameters were optimized using GridSearchCV, and after the models were trained, the root mean squared error (RMSE) was calculated based on fivefold cross-validation. Random forest regression is an ensemble method that works by having multitude of decision trees, where each is constructed independently from a different subsample ${ }^{52}$. Random forest regression resulted in the lowest RMSE, and this fitted model was used to rank the feature importance of the material properties and processing conditions using SHAP (SHapley Additive exPlanations) $)^{35}$. The SHAP formula is shown in Eq. (1), where $g$ is the explanation model, $M$ is the maximum coalition size/the number of simplified input features, $\phi_{i} \in \mathbb{R}$ is the feature attribution for a feature $i, z / \epsilon\{0,1\}^{M}$, and $\phi_{0}$ represents the model output with all the simplified inputs missing.

$$
g\left(z^{\prime}\right)=\phi_{0}+\sum_{i=1}^{M} \phi_{i} z_{i}^{\prime}
$$

Our data and trained models are available in the GitHub repository (https:// github.com/PV-Lab/capping-layer).

\section{Data availability}

The machine-learning input that supports the findings of this study are available in PubChem 2019 database: https://doi.org/10.1093/nar/gky1033.34 The degradation onset and slope data are available in Supplementary Data 1.

\section{Code availability}

The codes and the data sets used for preprocessing and regression are available in GitHub repository (https://github.com/PV-Lab/capping-layer). The regression algorithms are implemented using scikit-learn ${ }^{46}$ and the Shapley values are implemented using SHAP ${ }^{35}$ Python library.

Received: 20 January 2020; Accepted: 14 July 2020;

Published online: 20 August 2020

\section{References}

1. Jones-Albertus, R., Feldman, D., Fu, R., Horowitz, K. \& Woodhouse, M. Technology advances needed for photovoltaics to achieve widespread grid price parity. Prog. Photovolt. Res. Appl 24, 1272-1283 (2016).

2. National Renewable Energy Lab. Best research-cell efficiency chart. Photovoltaic Research. NREL. https://www.nrel.gov/pv/cell-efficiency.html. Accessed 21 October 2019.

3. Milot, R. L. et al. Charge-carrier dynamics in 2D hybrid metal-halide perovskites. Nano Lett. 16, 7001-7007 (2016).

4. Hartono, N. T. P. et al. The effect of structural dimensionality on carrier mobility in lead-halide perovskites. J. Mater. Chem. A https://doi.org/10.1039/ C9TA05241K (2019).

5. Herz, L. M. Charge-carrier dynamics in organic-inorganic metal halide perovskites. Annu. Rev. Phys. Chem. 67, 65-89 (2016).

6. Jung, E. H. et al. Efficient, stable and scalable perovskite solar cells using poly (3-hexylthiophene). Nature 567, 511-515 (2019).

7. Yoo, J. J. et al. An interface stabilized perovskite solar cell with high stabilized efficiency and low voltage loss. Energy Environ. Sci. 12, 2192-2199 (2019).

8. Gao, P., Bin Mohd Yusoff, A. R. \& Nazeeruddin, M. K. Dimensionality engineering of hybrid halide perovskite light absorbers. Nat. Commun. 9, 5028 (2018).

9. Lv, Y. et al. Bromine doping as an efficient strategy to reduce the interfacial defects in hybrid two-dimensional/three-dimensional stacking perovskite solar cells. ACS Appl. Mater. Interfaces 10, 31755-31764 (2018).

10. Wang, Y. et al. Efficient $a-\mathrm{CsPbI}_{3}$ photovoltaics with surface terminated organic cations. Joule 2, 2065-2075 (2018)

11. Schlipf, J. et al. Shedding light on the moisture stability of 3D/2D hybrid perovskite heterojunction thin films. ACS Appl. Energy Mater. 2, 1011-1018 (2019).
12. Wang, H. et al. Synergy of hydrophobic surface capping and lattice contraction for stable and high-efficiency inorganic $\mathrm{CsPbI}_{2} \mathrm{Br}$ perovskite solar cells. Sol. RRL 2, 1800216 (2018).

13. Dong, H. et al. Conjugated molecules "Bridge": functional ligand toward highly efficient and long-term stable perovskite solar cell. Adv. Funct. Mater. 29, 1808119 (2019).

14. Wang, F. et al. Phenylalkylamine passivation of organolead halide perovskites enabling high-efficiency and air-stable photovoltaic cells. Adv. Mater. 28, 9986-9992 (2016)

15. Wang, R. et al. Constructive molecular configurations for surface-defect passivation of perovskite photovoltaics. Science 366, 1509-1513 (2019)

16. Wang, R. et al. Caffeine improves the performance and thermal stability of perovskite solar cells. Joule 3, 1464-1477 (2019).

17. Lin, Y. et al. Enhanced thermal stability in perovskite solar cells by assembling 2D/3D stacking structures. J. Phys. Chem. Lett. 9, 654-658 (2018).

18. Koh, T. M. et al. Enhancing moisture tolerance in efficient hybrid 3D/2D perovskite photovoltaics. J. Mater. Chem. A 6, 2122-2128 (2018).

19. Zhou, Q. et al. High-performance perovskite solar cells with enhanced environmental stability based on a $\left(p-\mathrm{FC}_{6} \mathrm{H}_{4} \mathrm{C}_{2} \mathrm{H}_{4} \mathrm{NH}_{3}\right)_{2}\left[\mathrm{PbI}_{4}\right]$ capping layer. Adv. Energy Mater. 9, 1802595 (2019).

20. Luo, W. et al. Efficient and stable perovskite solar cell with high open-circuit voltage by dimensional interface modification. ACS Appl. Mater. Interfaces 11, 9149-9155 (2019)

21. Huang, X. et al. Enhanced efficiency and light stability of planar perovskite solar cells by diethylammonium bromide induced large-grain 2D/3D hybrid film. Org. Electron. Phys. Mater. Appl. 67, 101-108 (2019).

22. Feng, X. et al. Perfection of perovskite grain boundary passivation by Euporphyrin complex for overall-stable perovskite solar cells. Adv. Sci. 6, 1802040 (2019).

23. Kumar, J. N. et al. Machine learning enables polymer cloud-point engineering via inverse design. npj Comput. Mater. 5, 73 (2019).

24. Noh, J. et al. Inverse design of solid-state materials via a continuous representation. Matter https://doi.org/10.1016/j.matt.2019.08.017 (2019).

25. Zunger, A. Inverse design in search of materials with target functionalities. Nat. Rev. Chem. 2, 0121 (2018).

26. Dordević, N. et al. Machine learning for analysis of time-resolved luminescence data. ACS Photonics 5, 4888-4895 (2018).

27. Howard, J. M., Tennyson, E. M., Neves, B. R. A. \& Leite, M. S. Machine learning for perovskites' reap-rest-recovery cycle. Joule 3, 325-337 (2019).

28. Stoddard, R. J. et al. Forecasting the decay of hybrid perovskite performance using optical transmittance or reflected dark field imaging. ACS Energy Lett. https://doi.org/10.1021/acsenergylett.0c00164 (2020).

29. Onoda-Yamamuro, N., Matsuo, T. \& Suga, H. Calorimetric and IR spectroscopic studies of phase transitions in methylammonium trihalogenoplumbates (II)†. J. Phys. Chem. Solids 51, 1383-1395 (1990).

30. Menesatti, P. et al. RGB color calibration for quantitative image analysis: the "3D thin-plate spline" warping approach. Sensors 12, 7063-7079 (2012).

31. Spanopoulos, I. et al. Uniaxial expansion of the 2D Ruddlesden-Popper perovskite family for improved environmental stability. J. Am. Chem. Soc. 141, 5518-5534 (2019)

32. Hashmi, S. G. et al. Long term stability of air processed inkjet infiltrated carbon-based printed perovskite solar cells under intense ultra-violet light soaking. J. Mater. Chem. A 5, 4797-4802 (2017).

33. Hashmi, S. G. et al. Air processed inkjet infiltrated carbon based printed perovskite solar cells with high stability and reproducibility. Adv. Mater. Technol. 2, 1600183 (2017).

34. Kim, S. et al. PubChem 2019 update: improved access to chemical data. Nucleic Acids Res. 47, D1102-D1109 (2019).

35. Lundberg, S. M. \& Lee, S.-I. A unified approach to interpreting model predictions. Adv. Neural Inform. Process. 30, 4765-4774 (2017).

36. Mosconi, E. et al. Enhanced $\mathrm{TiO}_{2} / \mathrm{MAPbI}_{3}$ electronic coupling by interface modification with $\mathrm{PbI}_{2}$. Chem. Mater. 28, 3612-3615 (2016).

37. Aristidou, N. et al. Fast oxygen diffusion and iodide defects mediate oxygeninduced degradation of perovskite solar cells. Nat. Commun. 8, 1-10 (2017).

38. Fan, Z. et al. Layer-by-layer degradation of methylammonium lead tri-iodide perovskite microplates. Joule 1, 548-562 (2017)

39. Zheng, X. et al. Defect passivation in hybrid perovskite solar cells using quaternary ammonium halide anions and cations. Nat. Energy 2, 1-9 (2017).

40. Cheng, T. et al. Computation of octanol-water partition coefficients by guiding an additive model with knowledge. J. Chem. Inf. Model. 47, 2140-2148 (2007).

41. Hendrickson, J. B., Huang, P. \& Toczko, A. G. Molecular complexity: a simplified formula adapted to individual atoms. J. Chem. Inf. Model. 27, 63-67 (1987).

42. Bertz, S. H. The first general index of molecular complexity. J. Am. Chem. Soc. 103, 3599-3601 (1981).

43. Ertl, P., Rohde, B. \& Selzer, P. Fast calculation of molecular polar surface area as a sum of fragment-based contributions and its application to the prediction of drug transport properties. J. Med. Chem. 43, 3714-3717 (2000). 
44. Wang, R., Fu, Y. \& Lai, L. A new atom-additive method for calculating partition coefficients. J. Chem. Inf. Comput. Sci. 37, 615-621 (1997).

45. Yang, S. et al. Functionalization of perovskite thin films with moisture-tolerant molecules. Nat. Energy 1, 15016 (2016).

46. Pedregosa, F. et al. Scikit-Learn: Machine Learning in Python. Journal of Machine Learning Research 12, 2825-2830 (2011).

47. Altman, N. S. An introduction to kernel and nearest-neighbor nonparametric regression. Am. Stat. 46, 175-185 (1992).

48. Ho, T. K. The random subspace method for constructing decision forests IEEE Trans. Pattern Anal. Mach. Intell. 20, 832-844 (1998).

49. Friedman, J. H. Greedy function approximation: a gradient boosting machine. Ann. Stat. 29, 1189-1232 (2001).

50. Kingma, D. P. \& Ba, J. L. In 3rd International Conference on Learning Representations, ICLR 2015 - Conference Track Proceedings (International Conference on Learning Representations, ICLR, 2015).

51. Chang, C.-C. \& Lin, C.-J. LIBSVM: A Library for Support Vector Machines. ACM transactions on intelligent systems and technology (TIST). 2, 1-27 (2011).

52. Breiman, L. Random forests. Statistics 45, 1-33 (2001).

53. Tiihonen, A. et al. Critical analysis on the quality of stability studies of perovskite and dye solar cells. Energy Environ. Sci. 11, 730-738 (2018).

54. Wang, R. et al. A review of perovskites solar cell stability. Adv. Funct. Mater. 29, 1808843 (2019).

55. Song, Z. et al. Impact of moisture on photoexcited charge carrier dynamics in methylammonium lead halide perovskites. J. Phys. Chem. Lett. 9, 6312-6320 (2018).

56. Peng, C., Chen, J., Wang, H. \& Hu, P. First-principles insight into the degradation mechanism of $\mathrm{CH}_{3} \mathrm{NH}_{3} \mathrm{PbI}_{3}$ perovskite: light-induced defect formation and water dissociation. J. Phys. Chem. C 122, 27340-27349 (2018).

57. Zhu, Z. et al. Interaction of organic cation with water molecule in perovskite $\mathrm{MAPbI}_{3}$ : from dynamic orientational disorder to hydrogen bonding. Chem. Mater. 28, 7385-7393 (2016).

58. Müller, C. et al. Water infiltration in methylammonium lead iodide perovskite: fast and inconspicuous. Chem. Mater. 27, 7835-7841 (2015).

59. Nimens, W. J. et al. Understanding hydrogen bonding interactions in crosslinked methylammonium lead iodide crystals: towards reducing moisture and light degradation. Pathw. Angew. Chem. Int. Ed. 58, 13912-13921 (2019).

60. Beznosikov, B. V. \& Aleksandrov, K. S. Perovskite-like crystals of the ruddlesden-popper series. Crystallogr. Rep. 45, 792-798 (2000).

61. Stoumpos, C. C. et al. Ruddlesden-Popper hybrid lead iodide perovskite 2D homologous semiconductors. Chem. Mater. 28, 2852-2867 (2016).

62. Chang, Y.-H., Lin, J.-C., Chen, Y.-C., Kuo, T.-R. \& Wang, D.-Y. Facile synthesis of two-dimensional Ruddlesden-Popper perovskite quantum dots with fine-tunable optical properties. Nanoscale Res. Lett. 13, 247 (2018)

63. Sutanto, A. A. et al. In situ analysis reveals the role of $2 \mathrm{D}$ perovskite in preventing thermal-induced degradation in 2D/3D perovskite interfaces. Nano Lett. https://doi.org/10.1021/acs.nanolett.0c01271 (2020).

64. Tang, X. et al. Photoinduced degradation of methylammonium lead triiodide perovskite semiconductors. J. Mater. Chem. A 4, 15896-15903 (2016)

65. Sun, Q. et al. Role of microstructure in oxygen induced photodegradation of methylammonium lead triiodide perovskite films. Adv. Energy Mater. 7, 1700977 (2017).

66. Yager, K. G. GitHub - CFN-softbio/SciAnalysis: SciAnalysis is a set of Python scripts for batch processing of image data, including X-ray scattering detector images. https://github.com/CFN-softbio/SciAnalysis. Accessed 11 December 2019.

\section{Acknowledgements}

We thank Kevin Yager and Masafumi Fukuto (Brookhaven National Laboratory) for assistance in synchrotron-based characterization; Rafael Gomez-Bombarelli (MIT), JuanPablo Correa-Baena (Georgia Institute of Technology), Ki-Jana B Carter (MIT), and Betar Gallant (MIT), for discussions and input in initial phase of organics screening;
REN Zekun Danny and Siyu Isaac Parker TIAN (Singapore-MIT Alliance for Research and Technology) for discussion and input regarding machine-learning methods. This research used 11-BM (CMS) beamline of the National Synchrotron Light Source II, a U.S. Department of Energy (DOE) Office of Science User Facility operated for the DOE Office of Science by Brookhaven National Laboratory under Contract No. DE-SC0012704. Parts of this study were performed at the Harvard University Center for Nanoscale Systems (CNS), a member of the National Nanotechnology Coordinated Infrastructure Network (NNCI), which is supported by the National Science Foundation under NSF award no. 1541959. CNS is part of Harvard University. This work also made use of the MRSEC Shared Experimental Facilities at MIT, supported by the National Science Foundation under award number DMR-1419807. This work was supported by the National Science Foundation (NSF) SusChem Grant CBET-1605547 [N.T.P.H.]; Skoltech Grant 1913/R as part of the Skoltech NGP Program [N.T.P.H.]; TOTAL SA research grant funded through MITeI Sustng Mbr 9/08, RPP [J.T., C.B., Z.L., S.S.]; Alfred Kordelin Foundation and Svenska Tekniska Vetenskaps-akademien i Finland [A.T.]; U.S. Department of Energy (DOE) under Photovoltaic Research and Development (PVRD) program under Award No. DE-EE0007535 [Z.L., F.O.]; the Institute for Soldier Nanotechnology (ISN) Grant W911NF-13-D-0001, the National Aeronautics and Space Administration (NASA) Grant NNX16AM70H [J.J.Y.]; a MISTI-Spain research grant, and Research Mobility Program in the US of UPM [D.F.M.]

\section{Author contributions}

N.T.P.H. and J.T.: fabricated the films. N.T.P.H., J.T., C.B., J.J.Y., R.L., and S.S.: performed in-depth materials characterization. N.T.P.H., A.T., F.O., and C.B.: analyzed the degradation images data and performed machine-learning analysis. N.T.P.H., Z.L., D.F.M., and S.S.: interpreted experimental results. M.G.B., T.B., and S.S.: supervised the study. N.T.P.H., T.B., and S.S.: contributed to the writing the paper.

\section{Competing interests}

The authors declare no competing interests.

\section{Additional information}

Supplementary information is available for this paper at https://doi.org/10.1038/s41467 020-17945-4.

Correspondence and requests for materials should be addressed to T.B. or S.S.

Peer review information Nature Communications thanks Aron Walsh, Mohammad Nazeeruddin and the other, anonymous, reviewer(s) for their contribution to the peer review of this work. Peer reviewer reports are available.

Reprints and permission information is available at http://www.nature.com/reprints

Publisher's note Springer Nature remains neutral with regard to jurisdictional claims in published maps and institutional affiliations.

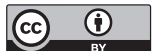

Open Access This article is licensed under a Creative Commons Attribution 4.0 International License, which permits use, sharing, adaptation, distribution and reproduction in any medium or format, as long as you give appropriate credit to the original author(s) and the source, provide a link to the Creative Commons license, and indicate if changes were made. The images or other third party material in this article are included in the article's Creative Commons license, unles indicated otherwise in a credit line to the material. If material is not included in the article's Creative Commons license and your intended use is not permitted by statutory regulation or exceeds the permitted use, you will need to obtain permission directly from the copyright holder. To view a copy of this license, visit http://creativecommons.org/ licenses/by/4.0/.

(C) The Author(s) 2020, corrected publication 2020 